\title{
A multiscale study on the morphology and evolution of slip bands in a nickel-based superalloy during low cycle fatigue
}

\author{
F. D. León-Cázares ${ }^{\mathrm{a}, *}$, R. Schlütter ${ }^{\mathrm{a}}$, T. Jackson ${ }^{\mathrm{b}}$, E. I. Galindo-Nava ${ }^{\mathrm{a}}$, C. M. F. Rae \\ ${ }^{a}$ Department of Materials Science and Metallurgy, University of Cambridge, 27 Charles Babbage Rd, \\ Cambridge CB3 OFS, UK \\ ${ }^{b}$ Rolls-Royce plc., Derby DE24 8BJ, UK
}

\begin{abstract}
Plastic deformation during low cycle fatigue in fcc materials with low stacking fault energy is accumulated in slip bands, which become preferential sites for crack initiation. Whilst these dislocation structures have been studied before, little has been done to assess the effect and evolution of the individual slip lines within them. In this study, samples of a $\gamma^{\prime}$ precipitate strengthened nickel-based superalloy are fatigued at room temperature and $700^{\circ} \mathrm{C}$ for 1,40 and 500 cycles. The resulting dislocation structures are characterised via Electron Channeling Contrast Imaging and Transmission Electron Microscopy. We introduce a new methodology to measure slip band parameters such as the slip line spacing and shear step length by analysing the holes left by sheared precipitates in $\gamma^{\prime}$-etched secondary electron micrographs. Statistics of these parameters are obtained and compared for different conditions. Advantages of this technique include resolution at the scale of individual planes, acquisition of true three-dimensional data and applicability in the bulk of the material. The combination of these techniques provides a unique mechanistic and quantitative insight into the slip band and precipitate morphology evolution.
\end{abstract}

Keywords: Plastic deformation; Slip band; Low cycle fatigue; Electron microscopy; Ni-based superalloys

\section{Introduction}

During low cycle fatigue, plastic deformation in nickel-based superalloys appears in the form of slip bands. Each band consists of a series of closely spaced parallel $\{111\}$ planes where dislocations accumulate and glide through the $\gamma$ matrix and $\gamma^{\prime}$ precipitates [1. The localisation of plastic strain in these planar structures is promoted by low stacking fault energies, high lattice friction stresses and the presence of short-range order [2 4], and is connected with fatigue damage and crack initiation [5] 7]. This multiscale study aims to answer some questions regarding the evolution of the slip bands and the accumulation of plastic deformation over many cycles.

*Corresponding author. Email: fdl22@cam.ac.uk

(c)2019. This manuscript version is made available under the CC-BY-NC-ND 4.0 
The sole activation of the Frank-Read sources explains only the formation of single slip lines, i.e. the intersection between an active slip plane and the sample surface. If no slip irreversibilities take place, the dislocation pile ups can annihilate when loading on reversal as seen by Pettinari-Sturmel during an in situ TEM analysis of a $\gamma$ single crystal [8]. A double cross slip process, originally proposed by Koehler [9], has been identified as the mechanism responsible for the lateral widening of the slip lines which ultimately results in the formation of the slip bands [10 13. According to this model, screw segments may cross slip and glide until the resultant forces again favour cross slip back onto the original plane orientation, forming a Koehler source [12, 14]. Further plastic straining generates additional dislocations on a new primary slip system close to the original. Loading on reversal may activate sources in the opposite direction, which eventually leads to the formation of the dipole structure typical of slip bands [10, 15].

The evolution of the slip bands is directly related to the development of slip steps at the surface of the material, which ultimately result in the formation of intrusions and extrusions [16. This topography shows only the number of dislocations that have reached the surface and their specific location and orientation, which is not enough to understand the complex interactions that happen at the grain interiors. To address this issue, numerous discrete dislocation dynamics analyses have been performed, mostly in precipitate-free materials. These have proven that cross slip is necessary for slip bands to form [14] and accumulate dislocation dipoles [10, although it is not clear how and where in the material this would occur for a more complex microstructure.

The behaviour of the individual slip lines at a smaller length scale is still unclear. The high dislocation densities that develop make it difficult to identify the active mechanisms via electron microscopy. The variability in the precipitate morphology and distribution of dipoles and multipoles characteristic of the slip bands [17, 18, likely affects the active slip planes in different proportions. A heterogeneous distribution of plastic slip has been observed in planar defects with digital image correlation (DIC) [19, 20, but the actual shear step lengths and slip line spacings are still unaccessible. Similarly, their effect on the evolution of the precipitate morphology has not been quantified.

The purpose of this work is to provide a deeper understanding of the formation and evolution of the slip bands prior to crack initiation. Interrupted tests of samples fatigued at two different temperatures are characterised via electron microscopy, in addition to using a newly developed technique that allows for the quantification of slip on individual planes.

\section{Material and methods}

The material examined in this study is the powder processed polycrystalline nickelbased superalloy RR1000, supplied by Rolls-Royce plc. The composition of this alloy is given in Table 1. The heat treatment consisted of a supersolvus solutioning at $1170^{\circ} \mathrm{C}$ for two hours, fan air cooling close to $-1^{\circ} \mathrm{C} / \mathrm{s}$, ageing at $760^{\circ} \mathrm{C}$ for 16 hours and static air cooling. Six dog bone shaped samples with a rectangular cross section of $11 \mathrm{~mm}$ x $5 \mathrm{~mm}$ were machined, and their $14 \mathrm{~mm}$ gauge lengths polished and shot peened to inhibit crack nucleation by inducing compressive stresses near the surface of the material. Note that all the characterisation techniques were performed in grains well over $200 \mu \mathrm{m}$ beneath the surface, where no considerable residual stresses from this process should exist [21]. 
Table 1: Chemical composition of alloy RR1000 (wt\%).

\begin{tabular}{ccccccccccc}
\hline $\mathrm{Ni}$ & $\mathrm{Cr}$ & $\mathrm{Co}$ & $\mathrm{Mo}$ & $\mathrm{Al}$ & $\mathrm{Ti}$ & $\mathrm{Ta}$ & $\mathrm{Hf}$ & $\mathrm{Zr}$ & $\mathrm{C}$ & $\mathrm{B}$ \\
\hline bal & 15 & 18.5 & 5 & 3 & 3.6 & 2 & 0.5 & 0.06 & 0.027 & 0.015 \\
\hline
\end{tabular}

Interrupted fatigue tests were performed at room temperature and $700^{\circ} \mathrm{C}$ for 1,40 and 500 cycles in an MTS servo-hydraulic test frame with a side contact extensometer and a furnace surrounding the specimen. A fully reversed strain control was used with a trapezoidal load waveform with an amplitude of $\Delta \epsilon / 2=0.6 \%$, strain rate of $0.5 \% \mathrm{~s}^{-1}$ and dwell times of one second at the minimum and maximum strains, starting with a tensile stage. The maximum and minimum stresses per cycle were recorded for each cycle, together with the cyclic stress-strain response.

SEM samples were cut parallel to the loading direction for every condition expecting some slip bands to be close to an edge-on orientation in preferentially aligned grains. Samples were also cut from undeformed material. All samples were hot mounted in bakelite, ground and polished with diamond suspensions and colloidal silica following standard procedures.

Electron Channeling Contrast Imaging (ECCI) was performed under the circular backscatter detector of a FEI NOVA NanoSEM. Multiple grains of each sample were imaged by tilting the stage within $\mathrm{a} \pm 1^{\circ}$ to get close to a two-beam condition. This detector was also used to measure the grain size via the circular intercept method as per the ASTM standard E112-13 with six circles of $700 \mu \mathrm{m}$ diameter.

A preferential $\gamma^{\prime}$ etchant detailed in reference [22] was applied to look at the morphology of sheared precipitates under the secondary electron through-lens detector of the SEM. Secondary and tertiary precipitates were independently imaged at $10 \mathrm{kX}$ and 50 $\mathrm{kX}$, respectively, by manually outlining the edges of the holes and performing the particle analysis in the Image J software package.

A slip band in a $\gamma^{\prime}$-etched sample appears as a series of holes sheared along parallel planes, as in Figure 1(a). The shear steps leave markings on the etched sample surface from which the orientation of slip can be deduced. The methodology detailed in Appendix A was used to access the true shear displacements produced at the scale of individual slip planes. This involves backtracking the consecutive angles the slip plane would need to be rotated, with reference to the initial configuration in Figure 1(b), to produce the shape of the hole observed once it has been etched away. The axes of the rotations by angles $\phi, \alpha$ and $\beta$ are the normal to the sample surface, the slip line projection and the normal to the slip plane, respectively. Figure 1(c-e) schematically shows the sequence of these rotations for a precipitate and a precipitate hole.

The angles are calculated by measuring characteristic lengths from the shear steps left in the etched holes; $\alpha$ is estimated from the elliptical projections of the circular sheared cross sections onto the specimen surface and $\beta$ from the sizes of the top and bottom halves of the precipitate holes. These are then used to estimate the real slip line spacings and shear step lengths. A statistical analysis of these parameters was carried out to understand the evolution of the plastic deformation behaviour within the slip bands.

This analysis considered the samples fatigued for 40 and 500 cycles in grains chosen at random. SEM micrographs with a resolution between 1 and $4.2 \mathrm{~nm} /$ pixel were used and all the measurements were taken between pixels with a similar intensity and a high 
(a)

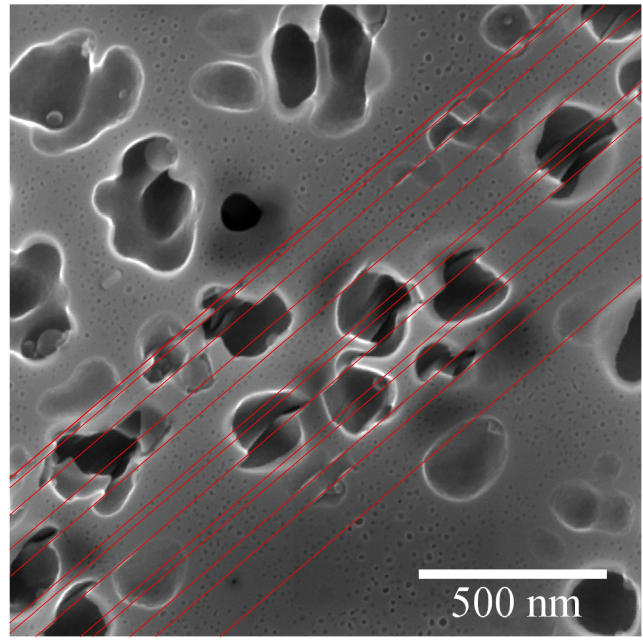

(b)

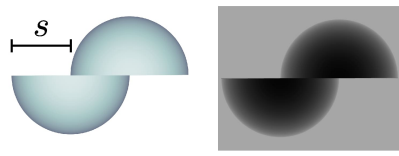

(c)
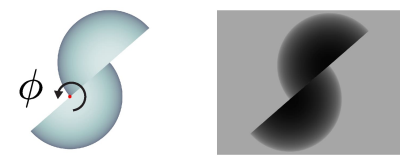

(d)
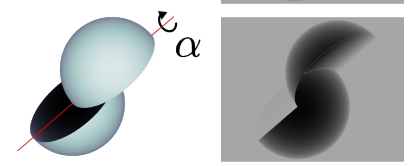

(e)

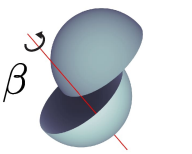

Figure 1: (a) Secondary electron micrograph of a $\gamma^{\prime}$-etched sample fatigued for 500 cycles at $20^{\circ} \mathrm{C}$ showing sheared precipitates. Red lines are drawn along the lines where the active slip planes intersect the sample surface. Models of a precipitate sheared and the hole it would leave in the matrix after being etched away are shown after consecutively applying (a) a shear step of length $s$ and rotations by angles (c) $\phi$, (d) $\alpha$ and (e) $\beta$.

brightness gradient. The loading direction was set horizontally in every micrograph to calculate the Schmid factor of the active slip system. The slip line spacings were measured once between every pair of slip markings, whereas the shear distances were recorded as the average of as many measurements as could be taken on multiple precipitate holes along a slip line, which typically varied between 1 and 6 . Most of these were done in the secondary $\gamma^{\prime}$ because the in- and out-of-plane shearing makes it harder to measure slip in the smaller tertiaries. When slip steps along the matrix/precipitate interface could be observed but they were smaller than a pixel and thus could not accurately be measured, they were recorded as the pixel size in the current micrograph.

Transmission electron microscopy (TEM) was also performed in specimens tested at $20^{\circ} \mathrm{C}$ and $700^{\circ} \mathrm{C}$ for 1 and 40 cycles to look at the dislocation interactions taking place within the slip bands. Slices of the material were cut parallel to the loading direction, spark eroded and electropolished with a solution of $10 \%$ perchloric acid in methanol. All the images were taken in a JEOL 200CX TEM in bright field mode.

\section{Results}

\subsection{Microstructure}

The material analysed has an ASTM grain size number of 6.15 (mean planar diameter of $43 \pm 4 \mu \mathrm{m}$ ), with multiple annealing twins. Rounded tertiariy precipitates populate the regions between the more flowery secondaries. Table 2 summarises the characterisation results for each precipitate family using the equivalent diameter $d_{e q}$ that would result in the same measured area for each particle. The circularity $C=4 \pi A / P^{2}$ (calculated from the individual areas $A$ and perimeters $P$ measured) is also given. 
Table 2: $\gamma^{\prime}$ characterisation.

\begin{tabular}{lcccc}
\hline Family & Count & Volume fraction $[\%]$ & Mean $d_{e q}[\mathrm{~nm}]$ & Circularity \\
\hline Secondary & 909 & 36.5 & 223 & 0.81 \\
Tertiary & 2774 & 5.7 & 10.8 & 0.96 \\
\hline
\end{tabular}

\subsection{Mechanical testing}

The LCF tests performed at each temperature gave similar results, with the sample loaded for 40 cycles at $20^{\circ} \mathrm{C}$ showing the highest variation in stress for the first cycle but still closely following the trend of the other curves, as seen in Figure 2(a). At both temperatures there is an initial hardening stage followed by cyclic softening. The peak stress occurs during the first 10 cycles at $20^{\circ} \mathrm{C}$ and between 20 and 40 cycles at $700^{\circ} \mathrm{C}$. Afterwards, the softening rate decreases in both cases but only at $20^{\circ} \mathrm{C}$ does it reach a saturation stage.

(a)

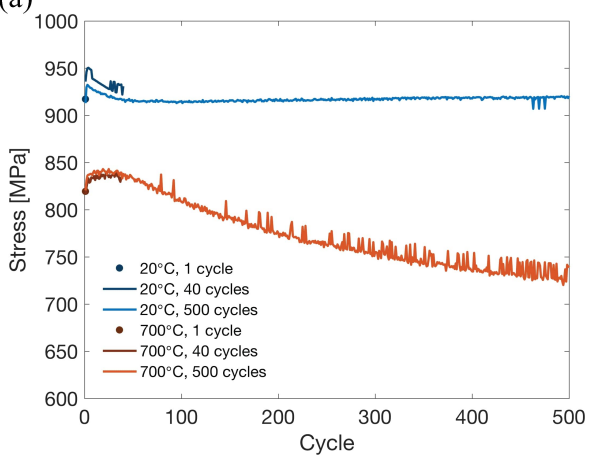

(b)

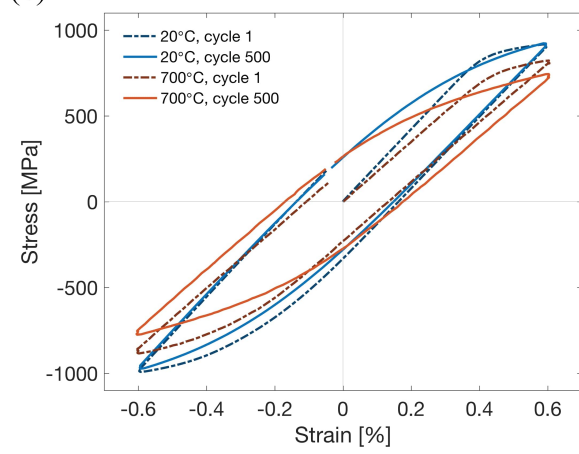

Figure 2: (a) Evolution of the maximum stress per cycle for the six samples tested in LCF and (b) first and last stress-strain hysteresis curves of the samples tested for 500 cycles at $20^{\circ} \mathrm{C}$ and $700^{\circ} \mathrm{C}$.

Figure 2(b) shows the hysteresis curves during the first and last cycles for each temperature. The plastic deformation experienced per cycle at different temperatures was similar when accounting for the hardening and softening regimes. After 500 cycles the cumulative plastic strain at $20^{\circ} \mathrm{C}$ is only $3.6 \%$ larger than that at $700^{\circ} \mathrm{C}$.

\section{3. $E C C I$}

The only sign of deformation in the undeformed sample is the presence of stacking faults on multiple slip planes. These have a length of a few micrometers and extend mostly through both matrix and precipitates, although in some instances their contrast disappears within secondary $\gamma^{\prime}$ particles. The density of stacking faults varies considerably between grains. No significant differences are observed between the stacking faults before and after deformation.

Slip bands appear as stripes with a higher intensity and they can be observed in all the deformed samples, as shown in Figure 3 . The slip bands in the primary slip system traverse the whole grain or the majority of it. Additionally, a clear contrast gradient 
can be seen along the length of many slip bands, indicating a variation in the dislocation density.
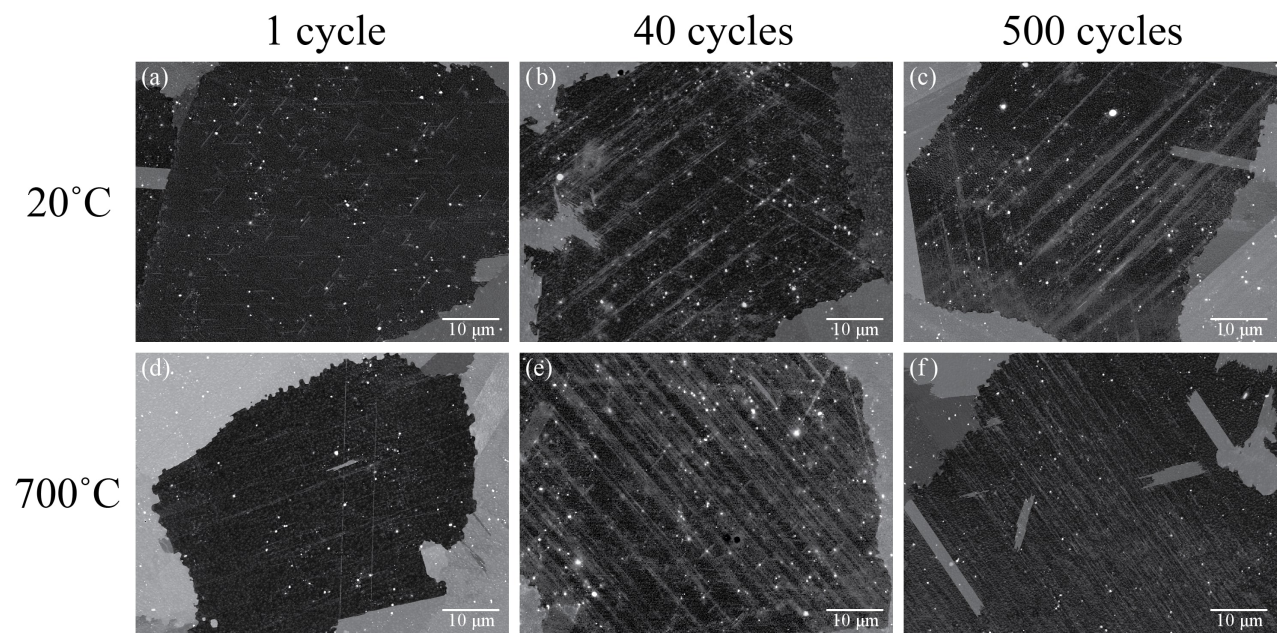

Figure 3: ECCI micrographs of samples fatigued at $20^{\circ} \mathrm{C}$ and $700^{\circ} \mathrm{C}$ for 1,40 and 500 cycles.

The deformation at room temperature is for the most part confined within slip bands. After just one cycle at room temperature, most grains show little or no deformation with ECCI. A few well defined slip bands with a spacing of over $5 \mu \mathrm{m}$ can be spotted within some grains, without any evidence of secondary slip. After 40 and 500 cycles, the material has more slip bands, which also look thicker and have a higher intensity. In some grains they appear in very small numbers, but in most cases multiple bands with the same orientation can be seen. Other grains show secondary slip and only in one case a third slip system contained a well defined slip band. Overall, there are no apparent differences between the deformation behaviour in both samples.

Cyclic deformation looks similar at $700^{\circ} \mathrm{C}$, but with a higher volume fraction of slip bands. The sample fatigued for one cycle has more grains where slip bands have formed. Upon further cyclic loading it once more becomes difficult to spot differences between the samples fatigued for 40 and 500 cycles. More slip bands appear in most grains, although some still show none. Secondary slip is scarcer at this temperature and no tertiary slip is observed. Instead, more and more slip bands develop along a single slip plane orientation in heavily deformed grains, to the point where parallel lines with a high contrast fill most of the area. An example of this more homogeneous deformation behaviour is seen in Figure 4 .

\subsection{Slip band shearing statistics}

Slip bands in $\gamma^{\prime}$-etched samples clearly show precipitate shearing. Shear steps are typically observed in all the precipitates along a slip line, but their lengths vary. Thus, reporting the average length per slip line gives a good indication of the overall behaviour at a section of the band rather than the measurements at individual precipitates.

The samples fatigued at both temperatures for one cycle show small amounts of shearing and slip bands are hard to spot. Three bands found for each condition show 

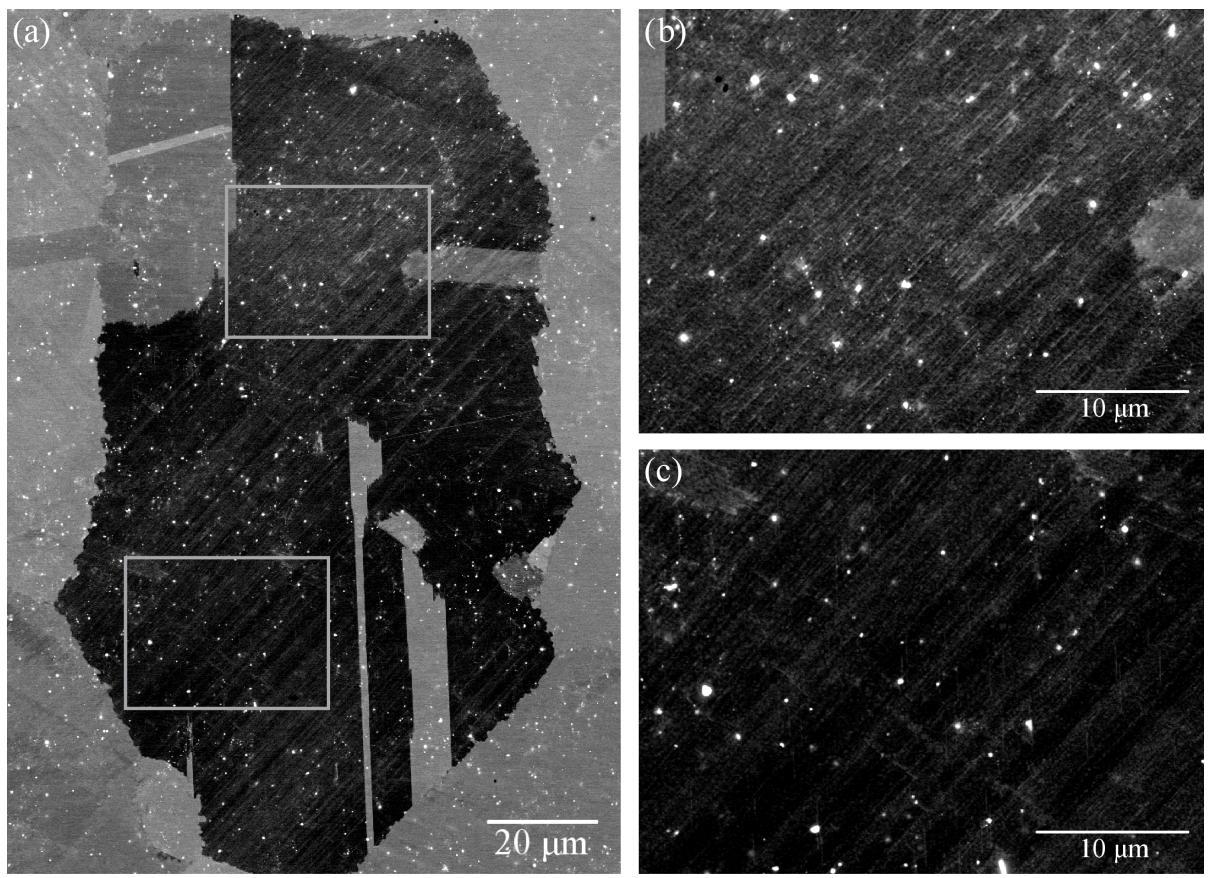

Figure 4: (a) ECCI micrograph of a grain with more homogeneous deformation from a sample fatigued at $700^{\circ} \mathrm{C}$ for 40 cycles. The regions inside the boxes are shown at a higher magnification in (b) and (c).

a maximum of four parallel slip lines. Although some slip bands form during the first fatigue cycle, as will be shown later by TEM, more cyclic deformation is required to promote precipitate shearing along most of their slip lines. No statistical analysis was performed in samples fatigued for one cycle due to the scarcity of slip bands.

The analysis was performed for a number of grains for each condition, accounting for one or more slip bands within each grain. Table 3 summarises the number of grains and slip bands characterised, as well as the number of measurements of slip line spacings $a$ and shear step lengths $s$.

Table 3: Number of features considered in the analysis. The slip line spacing $a$ and shear step length $s$ are defined in Figure A.16 and Figure 1 respectively.

\begin{tabular}{cccccc}
\hline Temperature & Number of cycles & Grains & $a$ measurements & $s$ measurements & Slip bands \\
\hline $20^{\circ} \mathrm{C}$ & 500 & 6 & 133 & 54 & 7 \\
& 40 & 4 & 69 & 44 & 4 \\
$700^{\circ} \mathrm{C}$ & 1 & 1 & 1 & 1 & 1 \\
& 500 & 3 & 103 & 104 & 13 \\
& 40 & 6 & 257 & 264 & $5^{*}$ \\
\end{tabular}

* Plus 3 regions of homogeneous deformation

In some cases it is not possible to access the true distances within a slip band due to variability in the precipitate morphology and experimental error. The measurements 
of $\alpha$ show an increase in its standard deviation $S D_{\alpha}$ towards larger angles due to the relatively low circularity of the secondary $\gamma^{\prime}$. For some orientations close to $\alpha=0^{\circ}$, i.e. the slip plane perpendicular to the specimen surface, the elliptical markings disappear as the minor radius becomes as small as the pixel size, in which case $S D_{\alpha}$ should be small $\left(<3^{\circ}\right)$ but is reported here as zero. Alternatively, the standard deviation $S D_{\beta}$ increases with bigger values of both $\alpha$ and $\beta$. The orientation of slip of the grains analysed altogether with the aforementioned trends can be seen in Figure 5 .

(a)

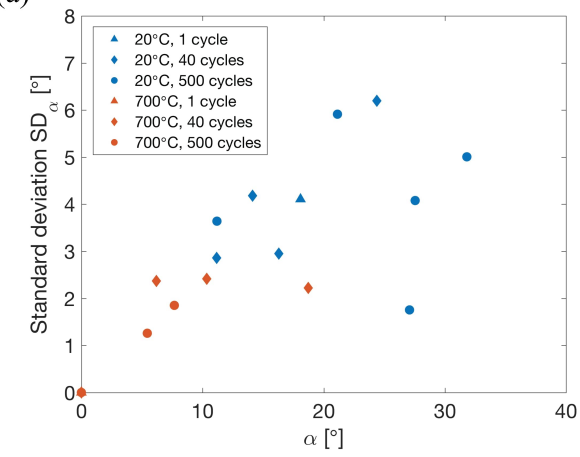

(b)

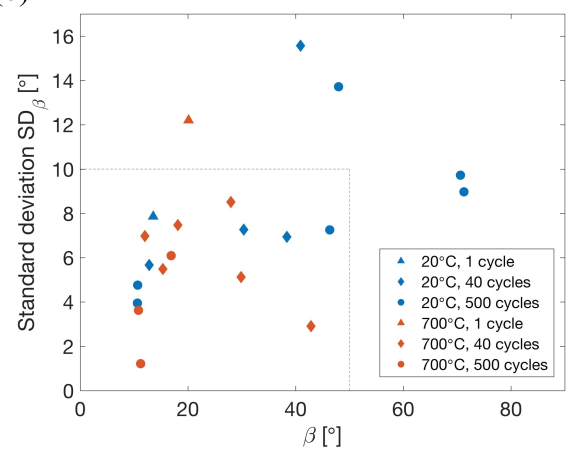

Figure 5: Angles (a) $\alpha$ and (b) $\beta$ and their standard deviations for the grains analysed. The samples outside the dashed box were ignored for the $s$-analysis. The angles $\alpha$ and $\beta$ are defined in Figure 1

The slip line spacings in all the grains analysed are considered in the present work, whereas the shear step lengths could not be measured for some crystal orientations. Values of $\beta$ further away from the ideal $\beta=0^{\circ}$ make the measurements of $s$ more difficult and inaccurate as their in- and out-of the viewing plane components grow bigger. Similarly, those grains where $S D_{\beta}>10^{\circ}$ cannot be properly measured. Thus, the grains that lie outside the dashed square in Figure 5(b) are not considered in the $s$-statistics.

The Schmid factors of the grains tested at both temperatures ranged between similar values. In total, $86 \%$ of the grains have a Schmid factor bigger than 0.4 , and the minimum value found is 0.3 . These are all within the range of the maximum Schmid factor for all possible orientations ( 0.27 to 0.5$)$. Moreover, no visible trends were found for any parameter as a function of the Schmid factor.

In some micrographs two or more nearby slip bands can be seen. However, it is not always clear where one ends and a new one begins. A limit is set here to an arbitrary value of $a=400 \mathrm{~nm}$, above which only 18 spacings were measured. Conversely, three of the grains analysed for the $700^{\circ} \mathrm{C}$ and 40 cycles sample show the presence of closely spaced slip lines throughout the whole micrograph, over a distance perpendicular to the slip plane of over $4 \mu \mathrm{m}$. These are regions of homogeneous deformation like those observed with ECCI. These grains are included in the following analysis except for the slip band width results.

The histograms of the slip line spacing in Figure 6(a) show no significant variation with the number of cycles at both temperatures. Alternatively, the active slip planes seem to be more spread out at higher temperatures. The average slip line spacings $\bar{a}$ at $20^{\circ} \mathrm{C}$ and $700^{\circ} \mathrm{C}$ are $45.3 \mathrm{~nm}$ and $74.6 \mathrm{~nm}$, respectively. These values consider all the slip line measurements performed at those temperatures for 40 and 500 cycles. 
(a)

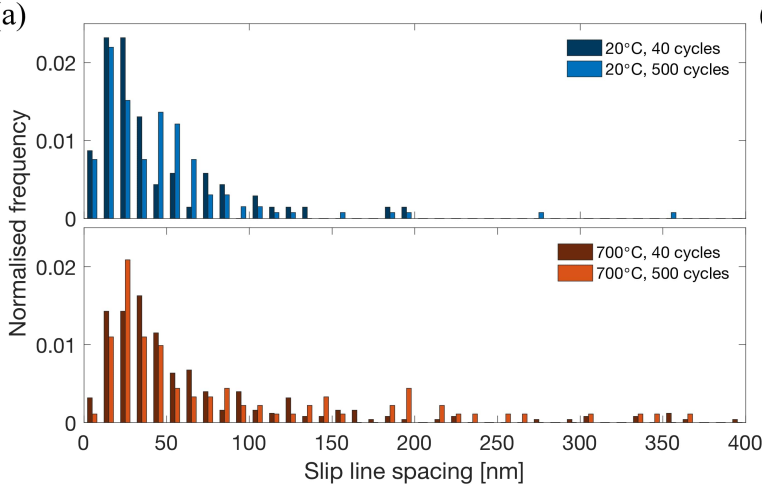

(b)

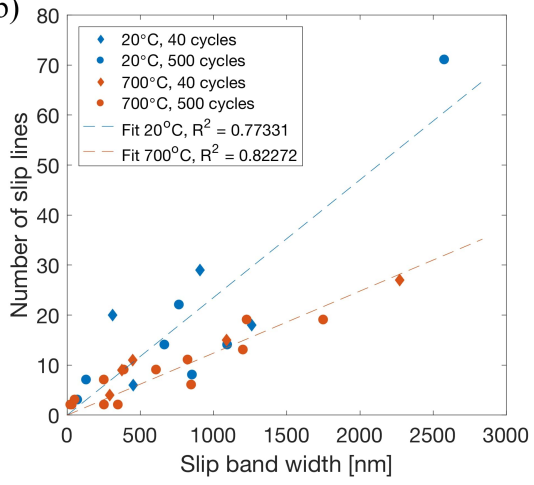

Figure 6: (a) Normalised histograms of the slip line spacings $a$ at $20^{\circ} \mathrm{C}$ and $700^{\circ} \mathrm{C}$ with bin widths of $10 \mathrm{~nm}$, accounting only for the values recorded lower than $400 \mathrm{~nm}$. (b) Plot of the number of slip lines each band contains as a function of the slip band width, with linear fits for each temperature.

Regarding the slip band width, not many measurements were taken due to the length scale at which the technique works, making it difficult to draw definitive conclusions. The values recorded are shown in Figure 6(b) together with the number of slip lines each band contained, showing again that the plastic deformation is more localised at $20^{\circ} \mathrm{C}$. The mean widths at $20^{\circ} \mathrm{C}$ and $700^{\circ} \mathrm{C}$ are 827 and $684 \mathrm{~nm}$, respectively. However, there is a big difference at room temperature between the widest band and the rest, and if this outlier was ignored a mean width of $652 \mathrm{~nm}$ would be obtained.

Most distributions of the shear step lengths within a slip band are centred around $s=0$, meaning that the combined effect of multiple slip lines within a slip band tends to neutralise. In about half of the grains the average shear distance per slip line is smaller than $2 \mathrm{~nm}$, and in the rest this value stays mostly under $4 \mathrm{~nm}$. An outlier, with a mean shear step length of $11 \mathrm{~nm}$, is actually one with homogeneous deformation. In this grain $88 \%$ of the slip lines show precipitates sheared in one direction.

By only measuring the shear step lengths it is not possible to know how many slip irreversibilities have taken place along each slip plane. These are events in which a dislocation glides in one direction but then is unable to glide back. Differt et al. [23] proposed a way of estimating the number of irreversibilities per slip line $2 z$ from the probability distribution $P$ of the number of slip steps $\Delta n=s / b$ when considering multiple active planes. The model assumes that the same number of dislocations glide along each slip line, as well as a history-independent probability for the generation of a slip irreversibility. This approach results in

$$
P(\Delta n)=\exp (-2 z) I_{\Delta n}(2 z)
$$

where $I_{\Delta n}$ is the modified Bessel function of the first kind. This equation is used here as the fitting function for the results of each condition shown in Figure 7)(a) in an effort to give a better insight into the physics of the precipitate shearing phenomenon. Note that $z$ and the mean shear step length $\overline{|s|}$ are related via equation

$$
\overline{|s|}=\underset{9}{2 b} \sqrt{\frac{z}{\pi}} .
$$


The reader is referred to the seminal work of Differt et al. 23] for further details on the derivation of equations (1) and (2).
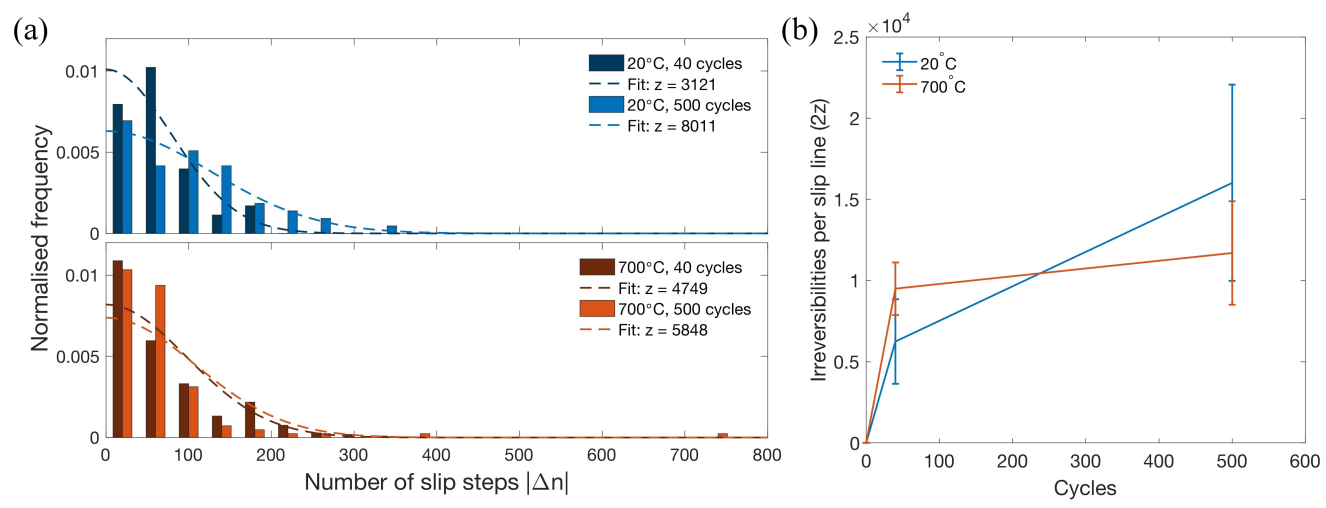

Figure 7: (a) Normalised histograms of the number of slip steps in each slip line at $20^{\circ} \mathrm{C}$ and $700^{\circ} \mathrm{C}$ with bin widths of 40 steps. The maximum likelihood estimation to a Bessel function of the first kind are shown in dashed lines for each condition. (b) Calculated number of slip irreversibilities as a function of the number of cycles, where the error bars correspond to the $95 \%$ confidence limits of the maximum likelihood estimates.

At both temperatures the estimated number of slip irreversibilities increases with more cycles as expected. Figure 7(b) shows that this evolution slows down after the first 40 cycles of the test. At a higher temperature the expected number of slip irreversibilities should be higher because of the increased cross slip activity. This behaviour is observed for the first 40 cycles, although the opposite is true after 500 cycles. This can be explained by accounting for the volume fraction of the material filled with slip bands, which is further discussed in Section 4

\subsection{TEM}

The TEM micrographs show the dislocation structures in more detail. In grains with small amounts of deformation, the predominant features observed are stacking faults a few microns long that extend through both phases, with some holes most likely around the precipitates. These types of defects were present in the material before the cyclic loading. It is possible that some of the stacking faults propagate upon loading as the partials bounding them may overcome glide obstacles, although the same stress would later be applied in the reverse direction. The question of how these stacking faults evolve during fatigue remains open.

The sample fatigued for one cycle at room temperature presents the smallest number of heavily deformed bands with a high dislocation density. In some instances only one or two nearby active slip planes extend over tens of microns, probably forming the precursors of more intensely deformed slip bands. Among these, dislocation loops and double loops are occasionally seen around secondary precipitates.

A thicker and more developed slip band in the same sample can be seen in Figure 8 (a). Even though the dislocation density varies abruptly within the single band, the same active slip planes for the most part can be seen on both sides of the dark region where the huge number of dislocations completely obscures the electron beam. This property is 
shared with the slip bands imaged for every condition. The slip lines sometimes become completely invisible in some regions but then show up again with the same spacing. This indicates that dislocations travel on such planes but only accumulate at some points where glide is impeded by obstacles.

(a)

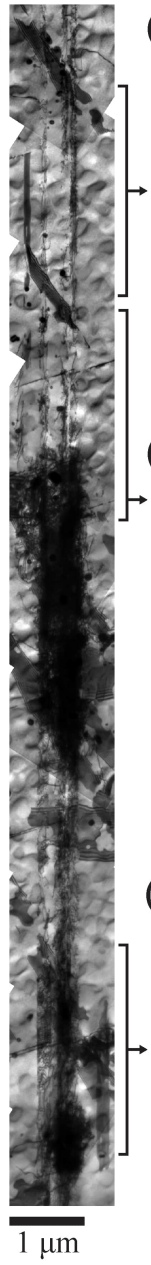

(b)

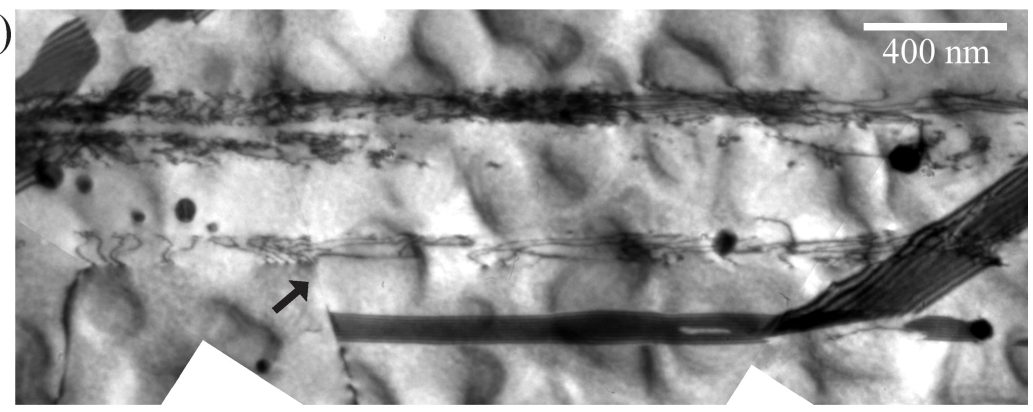

(c)

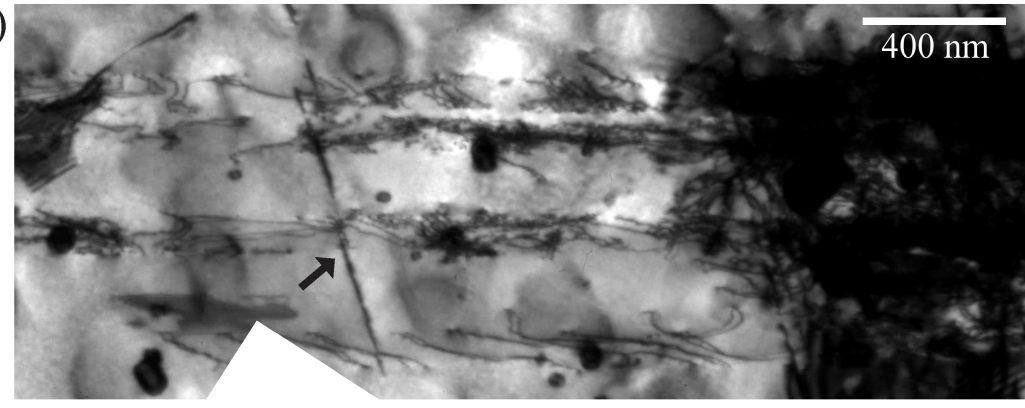

(d)

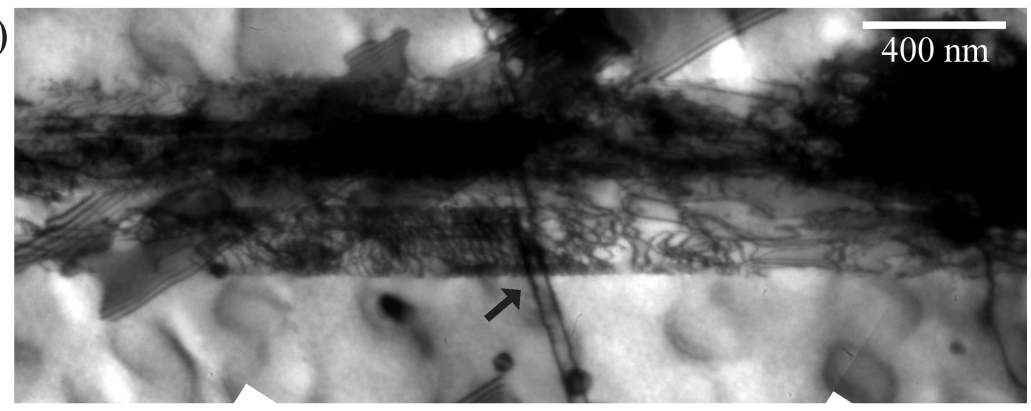

Figure 8: (a) TEM micrograph of a slip band from a sample fatigued at $20^{\circ} \mathrm{C}$ for 1 cycle. The areas indicated by the brackets are shown at higher magnifications and rotated $90^{\circ}$ counterclockwise in (b)-(d). The arrows point at interactions between dislocation pile ups in the slip band and non-coplanar edge-on defects.

Slip bands with a higher dislocation density over more slip planes do not contain dislocation loops, but rather show pile ups. With the accumulation of multiple dislocations in a single slip plane, the resulting high stresses may provoke the collapse of the loops. Furthermore, when these pile ups form in nearby slip planes they align with each other in such a way that the dislocations line up with each other to minimise the energy, as seen in Figure 8(d).

Non coplanar stacking faults appear to be the main obstacles against which pile ups 
initially form within the slip bands. Examples of these are shown in Figure 8(b)-(d). These barriers can however be overcome by some of the dislocations, which are then free to glide in the regions with a lower dislocation density. A change in the line vector of the dislocations that bypass the stacking fault is seen in many cases; e.g. in Figure 8(b). The intermittent contrast of these defects indicates the presence of dislocations along some regions of the faults.

Another feature that can be observed is the presence of cross slip in between the active planes of a slip band, as shown in Figure 9. This is additional evidence for double cross slip as the mechanism responsible for the thickening and intensification of the slip bands from the very beginning of the fatigue process.
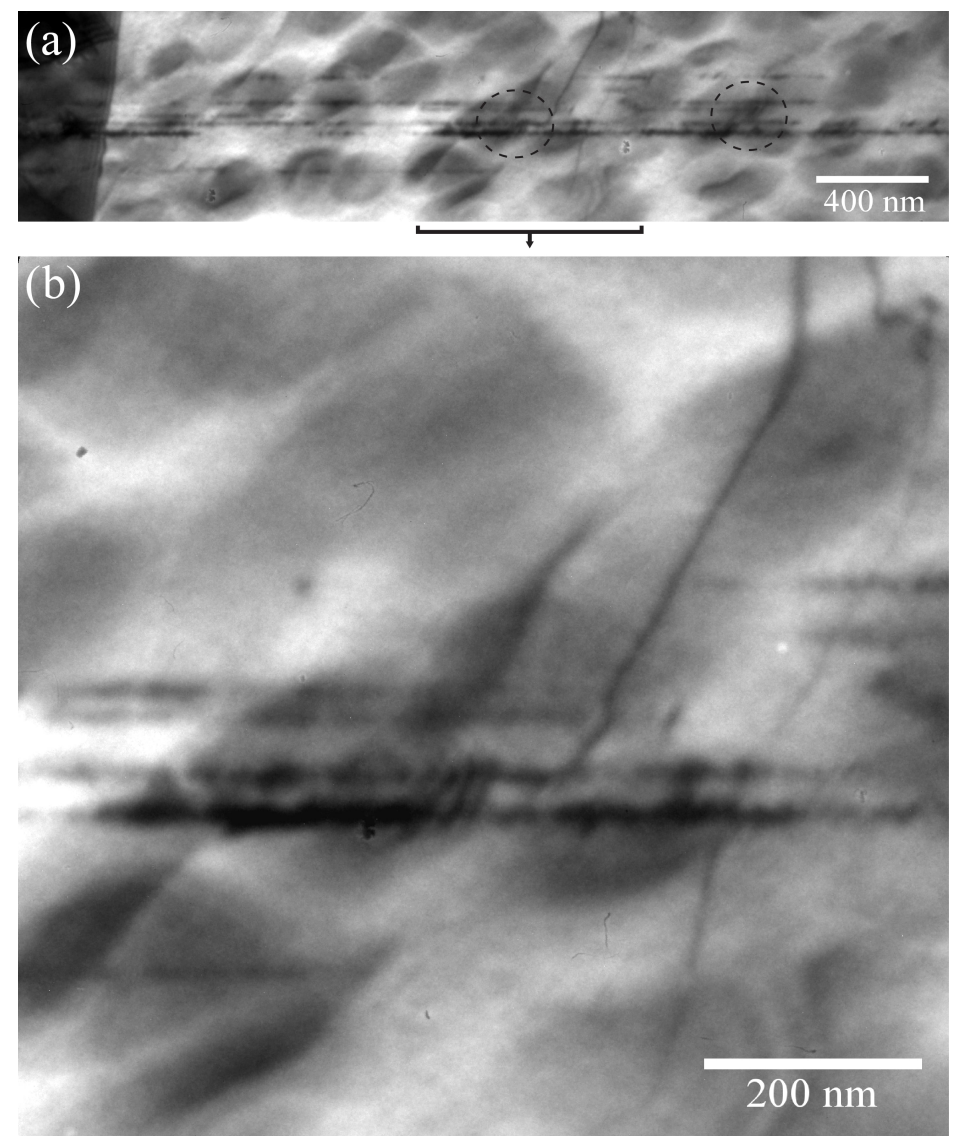

Figure 9: (a) TEM micrograph of an edge-on slip band from a sample fatigued at $20^{\circ} \mathrm{C}$ for 1 cycle where double cross slip events can be seen (enclosed by the dashed ellipses). The region indicated by the bracket is shown at a higher magnification in (b).

TEM in samples fatigued for 40 cycles at $20^{\circ} \mathrm{C}$ shows considerably more well developed slip bands. Some of the slip bands in this condition are heavily deformed and it becomes difficult to distinguish their features using this technique, as seen in Figure 10(a). Dark regions with a large dislocation density are seen along different sections of the slip band, mostly located at intersections with non-coplanar stacking faults and 
bands.

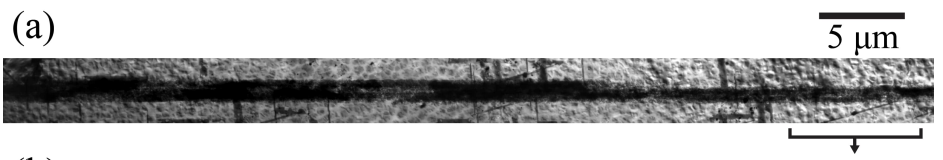

(b)

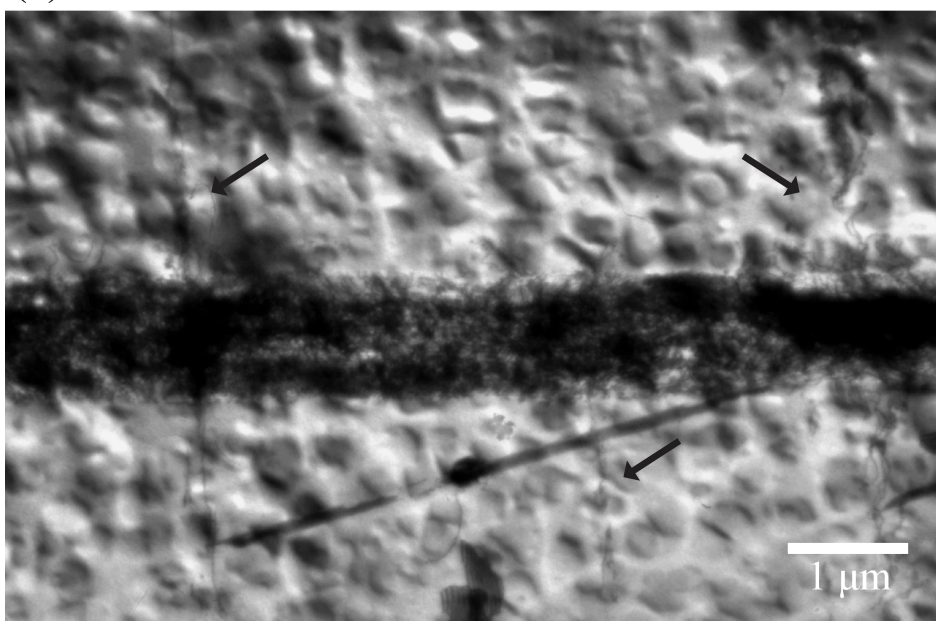

Figure 10: (a) TEM micrograph of a slip band from a sample fatigued at $20^{\circ} \mathrm{C}$ for 40 cycles. The region indicated by the bracket is shown at a higher magnification in (b). The arrows point towards secondary slip bands, only two of which have crossed the primary slip band.

Even though most of the deformation seems to be carried by a single slip plane orientation, secondary slip can often be seen in the form of very thin bands with only a few active slip planes that may or may not travel across the primary slip bands. This is shown at a higher magnification in Figure 10(b). In grains with preferential orientations or under higher strain amplitudes, these secondary slip bands may thicken and increase their dislocation density to form deformation structures such as that imaged with ECCI in Figures 3 (b) and (c).

More slip bands can be seen at $700^{\circ} \mathrm{C}$ after one cycle, compared to $20^{\circ} \mathrm{C}$. On average, these show activation of more slip planes, albeit with low dislocation densities. Similar features to those found at lower temperatures are seen, such as the presence of dislocation loops and the interactions between slip bands and non-coplanar stacking faults. Figure 11 shows an example of a slip band found in this condition. The formation of pile ups on nearby planes with aligned dislocations is similar to that described at $20^{\circ} \mathrm{C}$. The region within the slip band with the highest dislocation density is highlighted in Figure 11(c) by slightly coming off the two-beam condition used for Figure 11(b); this corresponds to the volume around the traversing stacking fault. Dislocations with a different Burgers vector are also present in this region.

After 40 cycles at $700^{\circ} \mathrm{C}$ the plastic deformation looks considerably less localised than at the lower temperature, in agreement with the slip line spacing statistical analysis. More slip bands populate the grain volume, but their dislocation densities never reach the levels seen at $20^{\circ} \mathrm{C}$. Figures 12 (a) and (b) show a slip band imaged under two different 
(a)
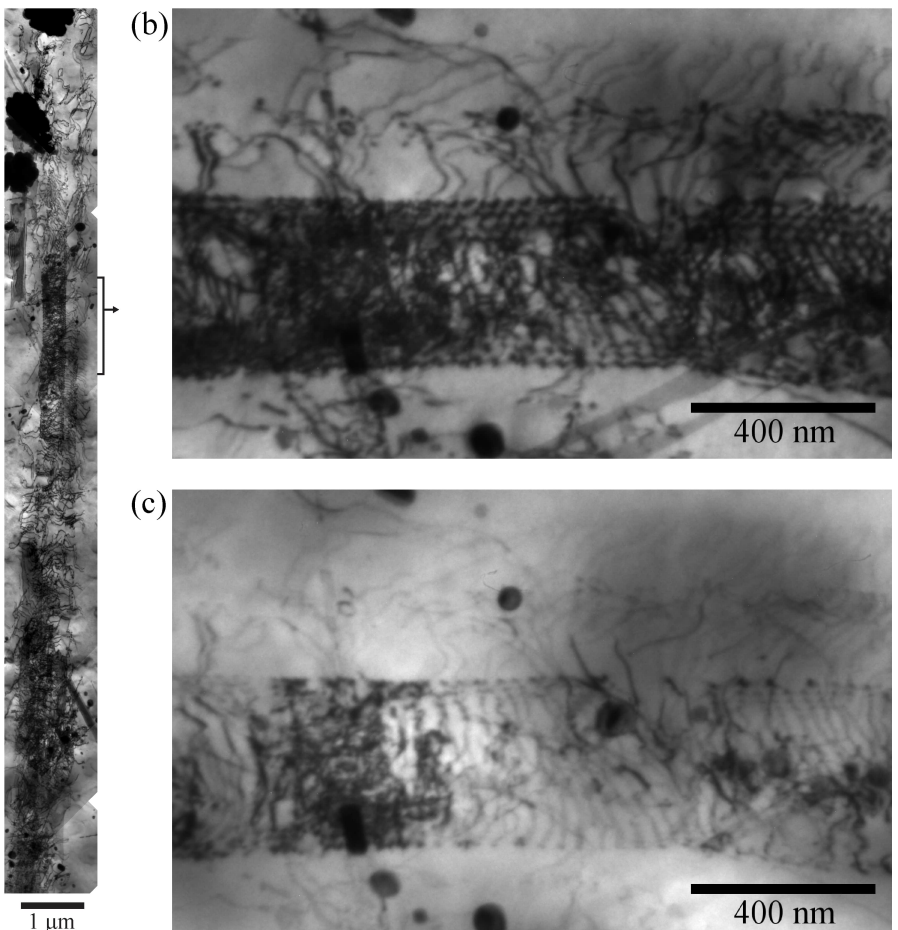

(c)

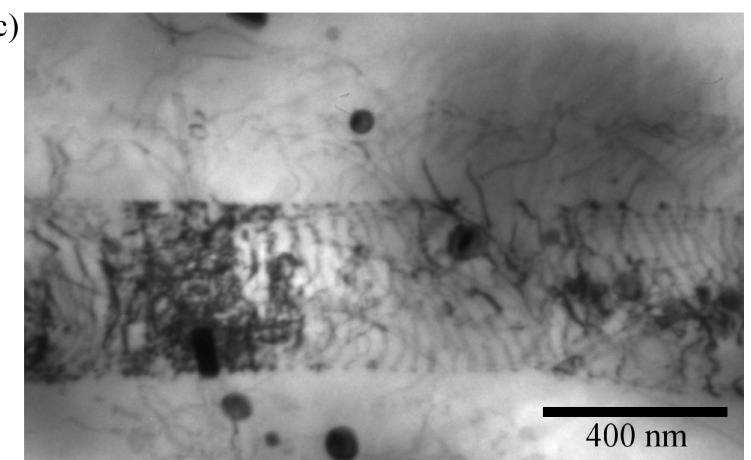

Figure 11: (a) TEM micrograph of a slip band from a sample fatigued at $700^{\circ} \mathrm{C}$ for 1 cycle. A region around a non-coplanar stacking fault is imaged (b) in a two-beam condition with a small positive deviation parameter and (c) with a larger positive deviation parameter.

two-beam conditions that suggest that most of the deformation is accommodated by a single slip system. Dislocations with a different Burgers vector are clear only close to the grain boundaries and to a lesser extent around the intersections between the slip bands and the non-coplanar stacking faults. This is to be expected as the stress states in these regions can vary considerably from that at the grain interior. This behaviour is also observed in neighbouring slip bands within the same grain.

Regions with more homogeneous deformation such as that in Figures 12(c) and (d) are also seen in this condition. The planarity of slip can be resolved in the form of multiple parallel active slip planes. Qualitatively, these dislocation structures look very similar to those of thinner slip bands. Note that narrow bands free of dislocations can still be seen in some regions of the material. This agrees with the observations performed with ECCI, and suggests that the plastic deformation may spread and cover the majority of a grain via the creation of new slip bands, the thickening of existing ones, or a combination of both. 

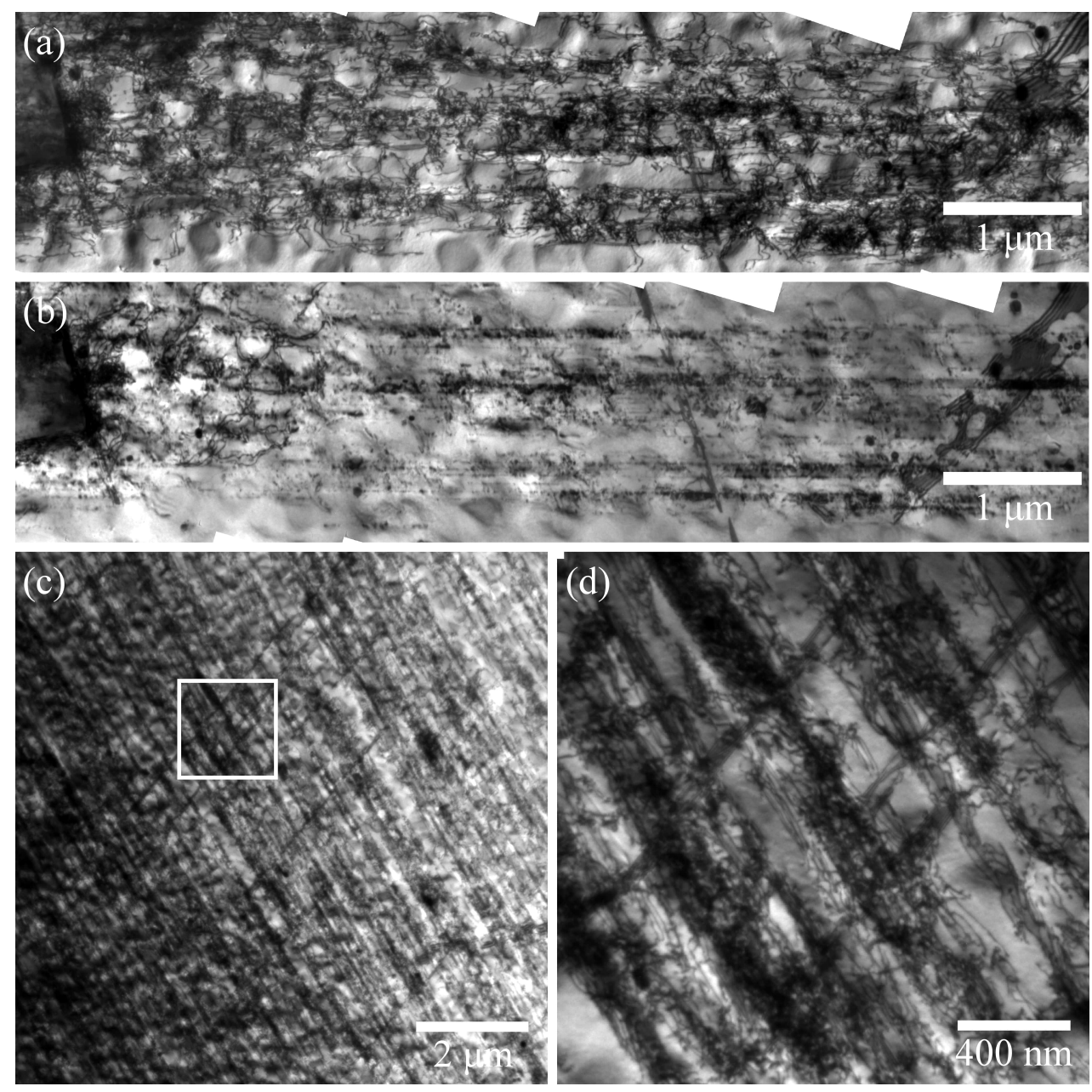

Figure 12: TEM micrographs of samples fatigued at $700^{\circ} \mathrm{C}$ for 40 cycles. (a)-(b) Single slip band imaged with two different beam conditions. (c) Micrograph of a grain with more homogeneous deformation, with the region inside the box shown at a higher magnification in (d).

\section{Discussion}

\subsection{Deformation behaviour}

Plastic deformation in RR1000 develops mainly in the form of slip bands. There are morphological differences between these bands at room temperature and $700^{\circ} \mathrm{C}$, but they show similar operating mechanisms and features. SEM in $\gamma^{\prime}$-etched samples and TEM reveal that most of the dislocations gliding within a slip band share the same Burgers vector and distribute heterogeneously along the active slip planes. The presence of stacking faults in similar number densities and sizes in samples with and without plastic deformation suggests these result from spontaneous dissociation during heat treatment and rules out a significant contribution of superlattice faults to plastic deformation. Dislocation climb was not observed either, and although it may appear during tests at 
$700^{\circ} \mathrm{C}$, its contribution is deemed inconsequential due to the planarity of slip observed in all the samples.

Plastic deformation in these conditions seems to be completely confined to the octahedral slip planes. A study by Mello et al. 24] showed that activation of cubic slip may occur during low cycle fatigue above $550^{\circ} \mathrm{C}$ in RR1000, and proposed a mechanism for alternating zig-zagged slip along $\{111\}$ cross slip planes in the matrix. No evidence of such dislocation behaviour is found in the present work. A possible cause for this is the lower strain amplitude imposed here, reason also for why secondary slip is scarce.

The sequence of the evolution of a slip band and the precipitates it shears is shown schematically in Figure 13 the individual steps in this sequence are discussed in detail in this section.

Any dislocation source may activate the initial slip plane. Orowan looping and shear via dislocation pairs are observed during the early stages of a slip band, but as the dislocation density increases the resulting stress results in the collapse of the loops. A similar phenomenon was identified by Brechet and Louchet 25] in a precipitate strengthened Al-Li alloy.

From the first cycle, double cross slip can result in the formation of Koehler sources [9] in neighbouring parallel planes a few nanometers apart, which results in an effective thickening of the band. Multiple dislocations in the cross slip plane may interconnect two or more slip lines, as shown in Figure 9.

A slip band will continue to thicken as more nearby slip planes become active via double cross slip until it reaches a steady state before 40 cycles, as negligible variation in slip band widths is seen between samples fatigued for 40 and 500 cycles. A low stacking fault energy typically results in thinner bands, but the widths obtained in this study are larger than most reported values in nickel-based superalloys $(<200 \mathrm{~nm})$ 17, 26. The largest secondary $\gamma^{\prime}$ precipitates in RR1000 are most likely responsible for this, as they are just smaller than the mean slip band widths. This may arise as a way for the material to accommodate more plastic deformation in the matrix around these.

The rate at which new slip bands form is not clear. It has been reported that in nickelbased superalloys these develop during the first cycle and further intensify with additional cyclic deformation [24, 27. However, the strain amplitudes used in those studies were higher than those in this work and others [28, 29, where a number of initial cycles are required to form the slip bands. The mobility of dislocations in the first slip bands may decrease as the dislocation density increases, becoming unable to accommodate plastic deformation in subsequent cycles. As a result, more slip bands would need to be formed during the early stages of the fatigue process.

Upon reaching the steady state, progressive precipitate degradation continues with the back-and-forth motion of dislocations. This is a consequence of mechanisms promoting slip irreversibility, e.g. the constant creation and annihilation of dislocations, cross slip events and the emergence of dislocations at a free surface 30 32. The accumulation of slip irreversibilities per slip line plotted in Figure 7 (b) increases monotonically with the number of cycles. However, large error bars arise from the number of measurements taken. Larger data sets giving a higher statistical significance would be required to draw decisive conclusions, but the current results give a strong indication of what is really happening in the material.

The main obstacle against which pile ups develop in this alloy are non-coplanar stacking faults. These hinder dislocation glide as they displace the slip plane by one third of 
(a)

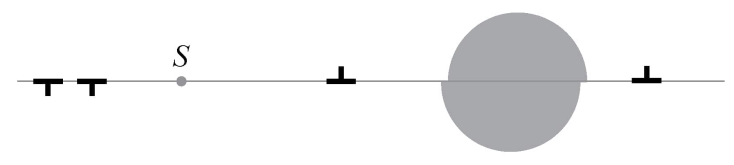

(b)

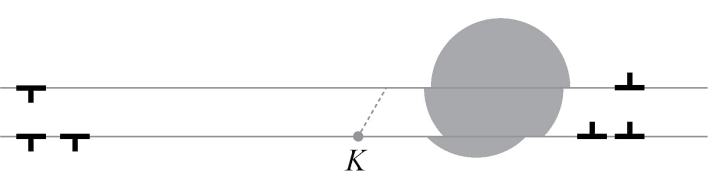

(c)

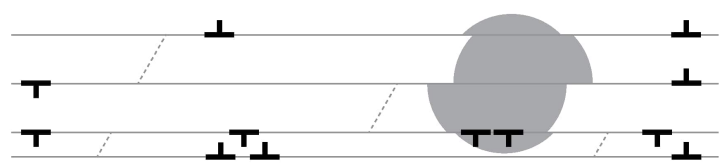

(d)

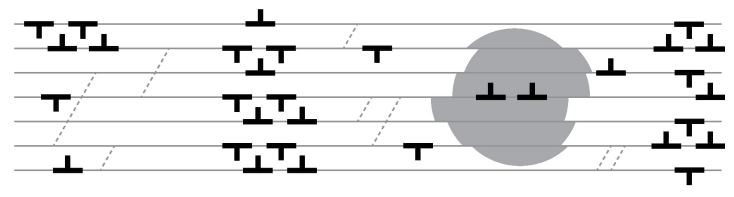

(e)

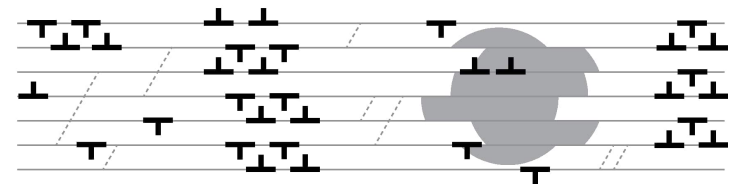

Figure 13: Schematic representation of the formation and evolution of a slip band and a precipitate. (a) Activation of the initial slip plane from a dislocation source $S$ and (b) generation of a Koehler source $K$ in a neighbouring plane via double cross slip during the first loading stage. Fatigue cycles produce (c) activation of more slip planes, slip band thickening and formation of dislocation multipoles until (d) a steady state in slip band width and dislocation density is reached. (e) Further cyclic loading results in continued degradation of the precipitates due to the back-and-forth motion of dislocations and slip irreversibilities.

the $d_{111}$ interplanar spacing. However, this lattice displacement may be overcome with a high enough stress such as that produced on the leading dislocation of a pile up, as shown in Figure 8(b). These defects act as semipermeable membranes where dislocations accumulate on many parallel slip planes within a slip band.

Referring to Thompson's tetrahedron [33, the character of a dislocation at the interface with such a fault may be either pure screw or mixed with a $60^{\circ}$ angle between 
its line and Burgers vectors. In the first scenario, shown in Figure 14(a), a dislocation may cross slip and either glide in the conjugate plane or cross slip back onto the primary orientation but on the other side of the stacking fault.
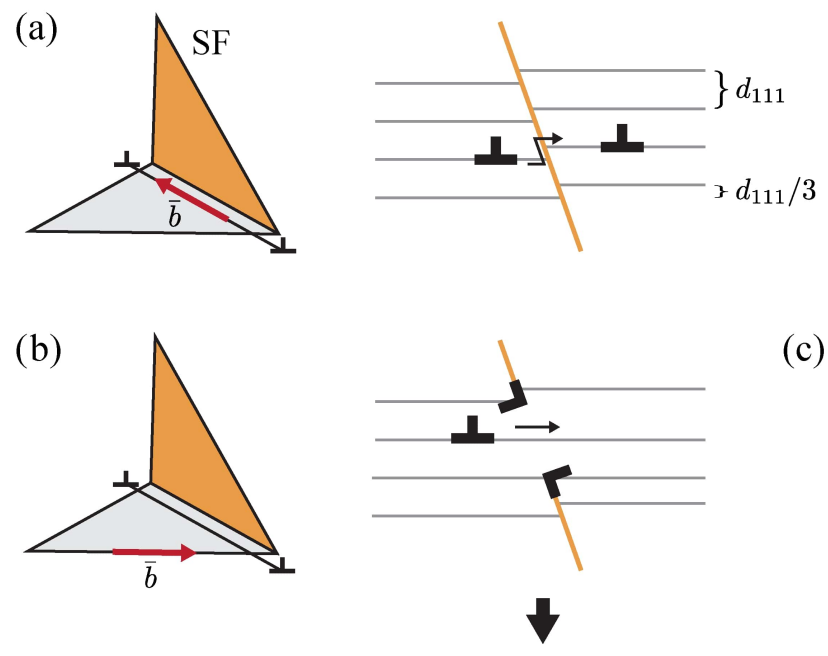

(c)
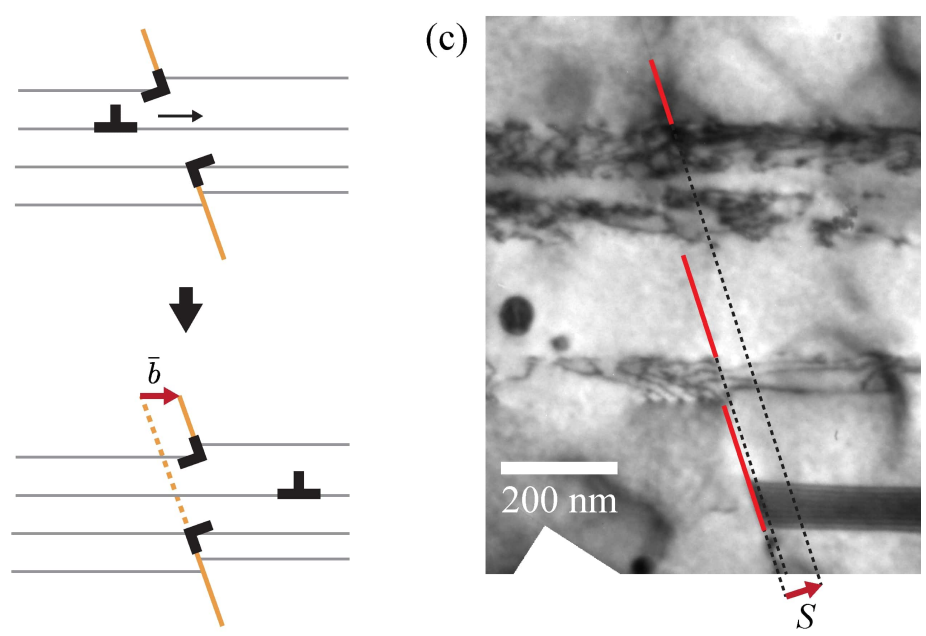

Figure 14: Two faces of Thompson's tetrahedron with the dislocation considered (left) and view along the line vector of the mechanisms proposed for non-coplanar stacking fault bypassing (right) for (a) a screw segment and (b) a dislocation with a $60^{\circ}$ character. (c) Region from TEM micrograph in Figure 8 (b) highlighting the stacking fault segments (red) and the shear displacement $S$ observed.

If the piling dislocations have a $60^{\circ}$ character instead, then climb would be needed to achieve the out-of-plane movement, requiring a large number of vacancies that may not be readily available at this strain rate and even less so at room temperature. Alternatively, a high stress may induce a realignment of the slip planes by the nucleation of partials on the conjugate plane with opposite Burgers vectors that locally remove the stacking fault, as illustrated in Figure 14(b). Upon glide through this narrow gap, one end of the stacking fault would be displaced by one Burgers vector. A misalignment of both ends would then only occur for the dislocations with an edge component. A closer look at the stacking faults from Figures 8(b) and (c) shows such displacements where dislocations have bypassed them, as highlighted for the former in Figure 14(c). The step length $S$ drawn in this micrograph must have been produced by $\sim 300$ dislocations that sheared the stacking fault.

Pile ups in nearby planes arrange themselves in structures with regular spacings, which is predicted by linear elasticity theory to occur only for dislocations with an edge character (pure screw segments would either cross slip and annihilate or just repel each 
other). Initially, these low energy dislocation structures such as that in Figure 8(d) are clearly not entirely formed of dislocation dipoles, as they have a net Burgers vector different from zero that makes them push against the stacking fault. Similarly, dislocations with an opposite Burgers vector may form these low energy structures on the other side of the stacking fault, as seen in Figure 11.

With more cycles these regions with a high dislocation density may develop more stable configurations as they accumulate dislocation dipoles and multipoles. Further plastic deformation is unlikely to occur in these regions as the mobility of the dislocations in them will be lower. This phenomenon is analogous to the classical theory of persistent slip bands, in which edge segments that manage to escape the rungs filled with dipoles bow out until they encounter the next obstacle and the deposited screw segments glide freely laterally [34. The main difference in this case is that the barriers are clear microstructural features with an irregular spacing against which the dislocations pile up, rather than self-forming structures composed of dipoles. This rationale may be extended in a similar way to interactions with non-coplanar slip bands, which will appear more frequently for higher strain amplitudes.

The main difference due to temperature spotted with the three techniques used is the distribution of slip lines. A larger separation between these is seen at $700^{\circ} \mathrm{C}$, which is rationalised here as a consequence of cross slip being a thermally activated mechanism. A similar argument as that used by Essmann and Mughrabi [35] suggests that below a critical separation in the direction normal to the slip plane, the screw segments will tend to cross slip and annihilate, further hindering plastic deformation. The orientation dependence of cross slip [36 38 may then be responsible in part for the scatter in the mean slip line spacings. Furthermore, for any single band there is also a variation in the spacings that arises from spatial variations in the precipitate distribution and the random nature of the double cross slip events during the formation process.

At a larger scale, this impacts directly on the fraction of material that develops slip bands. Having more widely spaced slip lines at $700^{\circ} \mathrm{C}$ implies that more regions in a grain must nucleate slip bands to accommodate the imposed macroscopic strain. Not developing bands in secondary slip systems further increases the need to form more slip bands in the primary system. The extreme cases observed of slip bands filling the totality of some grains exemplify this behaviour.

The calculated mean displacements per slip line show that overall the plastic strain left in the slip bands after the tests ended is relatively low. This is achieved by the combination of shear steps in opposite directions. The randomness of the sequence of shear steps is investigated by the probability of finding, for a given slip line with shear direction $\mathbf{n}_{\mathbf{s}}$, neighbours shearing the precipitates in the same direction. Ignoring the top and bottom slip lines of each slip band (and of each micrograph with homogeneous deformation), they can fall into three categories: (i) both neighbours shear in the same direction $\mathbf{n}_{\mathbf{s}}^{(\mathbf{1})}=\mathbf{n}_{\mathbf{s}}^{(\mathbf{2})}=\mathbf{n}_{\mathbf{s}}$, (ii) in the opposite direction $\mathbf{n}_{\mathbf{s}}^{(\mathbf{1})}=\mathbf{n}_{\mathbf{s}}^{(\mathbf{2})}=-\mathbf{n}_{\mathbf{s}}$ or (iii) they are opposite to each other $\mathbf{n}_{\mathbf{s}}^{(\mathbf{1})}=-\mathbf{n}_{\mathbf{s}}^{(\mathbf{2})}$, where $\mathbf{n}_{\mathbf{s}}^{(\mathbf{1})}$ and $\mathbf{n}_{\mathbf{s}}^{(\mathbf{2})}$ are the shearing directions of the neighbouring slip lines. As seen in Figure 15, categories (i) and (ii) cover around $25 \%$ of the cases each, which points towards a fully random behaviour. Deviations from this value appear towards larger shear step lengths, but this is attributed to the sample sizes being too small. The results for each category are (i) $23 \%$, (ii) $28 \%$ and (iii) $49 \%$. Thus, no obvious trend is observed and the clustering of slip lines in a specific pattern is 
ruled out.

(a)

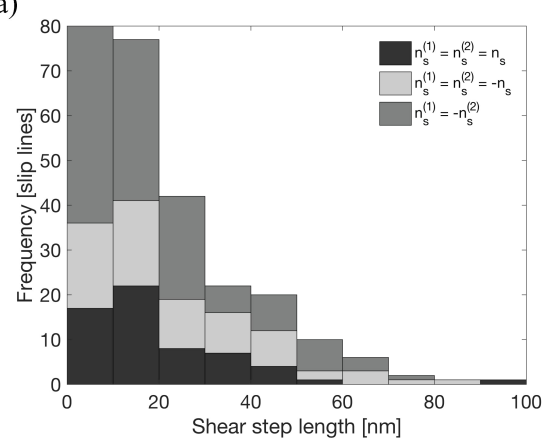

(b)

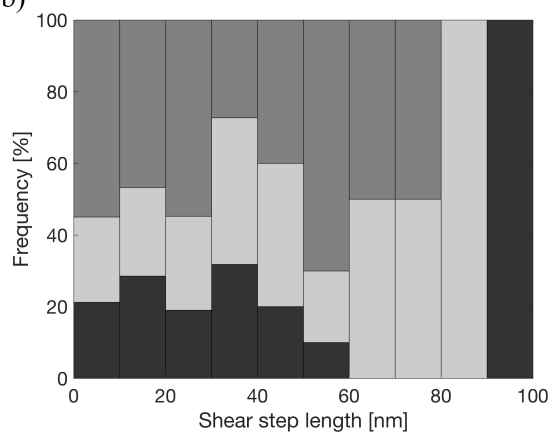

Figure 15: (a) Histogram of all the shear step lengths divided into three categories depending on the direction of shear of the neighbouring slip lines. The normalised results are plotted in (b).

The outlier grain with $88 \%$ of the slip lines pointing in one direction exhibits a very different behaviour. Even though the fatigue tests were interrupted after a compressive stage and thus some macroscopic plastic deformation is left within the material, it is unlikely that the shearing asymmetry arises only during the last loading stage because many of the lines would still show the previous shearing in both directions. One possible cause is that slip irreversibilities have a higher probability of occurring when loaded in tension than in compression (or vice versa), which would make shearing happen during that stage more likely to accumulate over many cycles. This again could happen for some loading orientations accounting for the probability of cross slip [37. Additional studies in slip reversibility are required.

\subsection{Slip line characterisation technique}

The methodology developed in this work proved valuable for accessing the true morphology of the slip bands. The resolution of the secondary electron through-lens detector in the SEM was able to capture shear step lengths as small as $1 \mathrm{~nm}$ long. The main source of error in the calculation of the slip orientation comes from the low sphericity of the secondary precipitates. This is specially true in the calculation of $\beta$ as it is more affected by deviations from a circular cross section.

The shear steps generate a series of extrusions and intrusions upon reaching the sample surface [16], which have been quantitatively studied before by means of atomic force microscopy (AFM) [28, 39,41] and high resolution digital image correlation (HRDIC) [20, 24, 42. The information extracted from these techniques is similar to that assessed in the current study, but with different limitations.

Advantages of the characterisation technique developed here are the higher resolution that allows for the quantification of slip at individual planes, the true three-dimensional data acquired and its applicability in the bulk of the material, affected neither by environmental effects nor image stresses at the free surface. AFM enables the quantification and measurement of steps, but at the surface of the material where image stresses are expected to alter the deformation behaviour. Also, this technique does not consider the direction of the displacements, i.e. the Burgers vector of the relevant slip system. 
HR-DIC cannot capture the behaviour of plastic deformation at the nanometre length scale as it assumes a continuous strain tensor rather than localised slip at individual planes. These discontinuities have recently been incorporated into a Heaviside-DIC [43, but its resolution is still lower than that achieved in this work. HR-DIC at high temperatures has been performed in nickel-based superalloys by using a stable oxide layer as the speckle pattern to track the deformation [20, but the evolution of such layer under some conditions limits its applicability.

Disadvantages of the introduced technique include its low speed and manual recording of the measurements taken, as well as its limitation to alloys with large precipitates. This could be improved by automating the task via image processing, but the contrast variability in a micrograph makes this difficult, especially for the calculation of $\alpha$ and $\beta$. Determining these angles could be facilitated by performing electron backscattered diffraction (EBSD) prior to applying the $\gamma^{\prime}$ etchant. Slip line spacings and shear step lengths can then be measured for precipitates as small as $20 \mathrm{~nm}$.

\section{Conclusions}

The current work provides a deeper understanding of the low cycle fatigue deformation behaviour at low and intermediate temperatures in polycrystalline nickel-based superalloys. Interrupted fatigue tests provide an insight into the evolution of this process from the beginning of the cyclic loading. The material characterisation is achieved by complementing a series of electron microscopy techniques:

1. ECCI provides an overview of the spatial variability in the deformation mechanisms that appear along multiple grains for each condition.

2. Secondary electron SEM in $\gamma^{\prime}$-etched samples is incorporated into a new methodology to quantify slip line spacings and shear step lengths at the nanometre length scale.

3. Bright field TEM is used to characterise the specific dislocation structures and mechanisms responsible for cyclic plastic deformation.

The low stacking fault energy and large precipitates restrain the plastic deformation mostly to slip bands that start developing from the first cycle. Unlike in other nickelbased superalloys, the slip bands have here similar thicknesses at both temperatures. Features in these dislocation structures include extended slip lines with heterogeneous dislocation densities, dislocation dipoles and multipoles, pile ups at non-coplanar stacking faults and double cross slip events. A sound explanation for the heterogenisation of the dislocation density within the slip bands is given as a consequence of their interaction with non-coplanar defects, which act as permeable membranes to dislocation glide.

At room temperature the plastic deformation in the slip bands is more localised, due to shorter slip line spacings and higher dislocation densities. Cross slip as a dislocation annihilation mechanism for slip in nearby planes fully explains this behaviour. At higher temperatures the lower local plastic strain attained forces more slip bands to develop across the grains. For the $0.6 \%$ strain amplitude used here, secondary slip is scarcer at $700^{\circ} \mathrm{C}$, which contributes to the formation of more bands in the primary slip system, to the point where they may get too close and fill the entirety of some grains. Furthermore, the only quantitative difference found between the slip bands in samples fatigued for 40 
and 500 cycles is the average shear step length per slip line, which increases as the test continues.

\section{Acknowledgements}

F.D. León-Cázares is grateful for funding from CONACyT, the Cambridge Trust and the Roberto Rocca Education Program. E.I. Galindo-Nava acknowledges funding from RAEng for his research fellowship. We also acknowledge Rolls-Royce plc and the Engineering and Physical Sciences Research Council (EPSRC) for financial support under the Strategic Partnership, Grant Numbers EP/ H022309/1 and EP/H500375/1, and thank Rolls-Royce plc for supplying the material and performing the mechanical testing.

\section{References}

[1] R. Stoltz, A. Pineau, Dislocation-precipitate interaction and cyclic stress-strain behavior of a $\gamma^{\prime}$ strengthened superalloy Mater. Sci. Eng. 34 (3) (1978) 275-284.

[2] F. Pettinari, J. Douin, G. Saada, P. Caron, A. Coujou, N. Cle, Stacking fault energy in short-range ordered gamma-phases of Ni-based superalloys, Mater. Sci. Eng. A 325 (2002) 511-519.

[3] A. Pineau, S. D. Antolovich, High temperature fatigue of nickel-base superalloys - A review with special emphasis on deformation modes and oxidation Eng. Failure Anal. 16 (8) (2009) 2668-2697.

[4] S. Mahajan, Critique of mechanisms of formation of deformation, annealing and growth twins: Face-centered cubic metals and alloys Scr. Mater. 68 (2) (2013) 95-99.

[5] B. A. Lerch, N. Jayaraman, S. D. Antolovich, A study of fatigue damage mechanisms in Waspaloy from 25 to 800C, Mater. Sci. Eng. 66 (2) (1984) 151-166.

[6] H. T. Pang, P. A. S. Reed, Fatigue crack initiation and short crack growth in nickel-base turbine disc alloys - The effects of microstructure and operating parameters, Int. J. Fatigue 25 (9-11) (2003) 1089-1099.

[7] J. Stinville, N. Vanderesse, F. Bridier, P. Bocher, T. Pollock, High resolution mapping of strain localization near twin boundaries in a nickel-based superalloy Acta Mater. 98 (2015) 29-42.

[8] F. Pettinari-sturmel, M. Jouiad, H. O. K. Kirchner, N. Clément, A. Coujou, Local disordering and reordering phenomena induced by mobile dislocations in short-range-ordered solid solutions, Philos. Mag. A 82 (16) (2002) 3045-3054.

[9] J. S. Koehler, The Nat. of Work-Hardening Phys. Rev. 86 (1) (1952) 52-59.

[10] C. Déprés, C. Robertson, M. Fivel, Crack initiation in fatigue: experiments and three-dimensional dislocation simulations, Mater. Sci. Eng. A 387-389 (1-2 SPEC. ISS.) (2004) 288-291.

[11] U. Messerschmidt, M. Bartsch, Generation of dislocations during plastic deformation, Mater. Chem. Phys. 81 (2-3) (2003) 518-523.

[12] C. Déprés, C. F. Robertson, M. C. Fivel, Low-strain fatigue in AISI 316L steel surface grains: a three-dimensional discrete dislocation dynamics modelling of the early cycles I. Dislocation microstructures and mechanical behaviour, Philos. Mag. 84 (22) (2004) 2257-2275.

[13] Z. Wang, I. Beyerlein, R. LeSar, Slip band formation and mobile dislocation density generation in high rate deformation of single fcc crystals, Philos. Mag. 88 (9) (2008) 1321-1343.

[14] A. M. Hussein, S. I. Rao, M. D. Uchic, D. M. Dimiduk, J. A. El-Awady, Microstructurally based cross-slip mechanisms and their effects on dislocation microstructure evolution in fcc crystals, Acta Mater. 85 (2015) 180-190.

[15] Y. Li, C. Laird, Cyclic response and dislocation structures of AISI 316L stainless steel. Part 2: polycrystals fatigued at intermediate strain amplitude Mater. Sci. Eng. A 186 (1-2) (1994) 87-103.

[16] U. Essmann, U. Gösele, H. Mughrabi, A model of extrusions and intrusions in fatigued metals I. Point-defect production and the growth of extrusions, Philos. Mag. A 44 (2) (1981) 405-426.

[17] H. Ho, M. Risbet, X. Feaugas, On the unified view of the contribution of plastic strain to cyclic crack initiation: Impact of the progressive transformation of shear bands to persistent slip bands Acta Mater. 85 (2015) 155-167.

[18] M. Petrenec, K. Obrtlík, J. Polák, Inhomogeneous dislocation structure in fatigued INCONEL 713 LC superalloy at room and elevated temperatures, Mater. Sci. Eng. A 400-401 (1-2 SUPPL.) (2005) 485-488. 
[19] T. Edwards, F. Di Gioacchino, H. Springbett, R. Oliver, W. Clegg, Stable Speckle Patterns for Nano-scale Strain Mapping up to 700 C Exp. Mech. 57 (9) (2017) 1469-1482.

[20] J. C. Stinville, M. P. Echlin, P. G. Callahan, V. M. Miller, D. Texier, F. Bridier, P. Bocher, T. M. Pollock, Measurement of Strain Localization Resulting from Monotonic and Cyclic Loading at 650 C in Nickel Base Superalloys, Exp. Mech. 57 (8) (2017) 1289-1309.

[21] O. M. Messé, S. Stekovic, M. C. Hardy, C. M. F. Rae, Characterization of Plastic Deformation Induced by Shot-Peening in a Ni-Base Superalloy, JOM 66 (12) (2014) 2502-2515.

[22] M. Preuss, P. J. Withers, J. W. L. Pang, G. J. Baxter, Inertia welding nickel-based superalloy: Part I. Metallurgical characterization. Metall. Mater. Trans. A 33 (10) (2002) 3215-3225.

[23] K. Differt, U. Essmann, H. Mughrabi, Models of particle destruction in fatigued precipitationhardened alloys Phys. Status Solidi (a) 104 (1) (1987) 95-106.

[24] A. W. Mello, A. Nicolas, M. D. Sangid, Fatigue strain mapping via digital image correlation for Ni-based superalloys: The role of thermal activation on cube slip Mater. Sci. Eng. A 695 (December 2016) (2017) 332-341.

[25] Y. Brechet, F. Louchet, On the stability of orowan loops around coherent spherical ordered precipitates Acta Metall. 37 (9) (1989) 2469-2473.

[26] M. Risbet, X. Feaugas, Some comments about fatigue crack initiation in relation to cyclic slip irreversibility Eng. Fract. Mech. 75 (11) (2008) 3511-3519.

[27] M. Petrenec, J. Polák, T. Samoril, J. Dluhos, K. Obrtlík, In-Situ High Temperature Low Cycle Fatigue Study of Surface Topography Evolution in Nickel Superalloys, in: METAL 2014 Int. Conf. Metal. Mater.,23rd, Brno, 2014, pp. 1139-1144.

[28] M. Risbet, X. Feaugas, C. Guillemer-Neel, M. Clavel, Use of atomic force microscopy to quantify slip irreversibility in a nickel-base superalloy, Scr. Mater. 49 (6) (2003) 533-538.

[29] D. Khireddine, R. Rahouadj, M. Clavel, Irreversibility of strain during low-cycle fatigue experiments of a precipitation-hardened alloy Philos. Mag. A 77 (6) (1998) 1555-1566.

[30] H. Mughrabi, Cyclic Slip Irreversibilities and the Evolution of Fatigue Damage Metall. Mater. Trans. B $40(4)(2009)$ 431-453.

[31] H. Mughrabi, Cyclic slip irreversibility and fatigue life: A microstructure-based analysis, Acta Mater. 61 (4) (2013) 1197-1203.

[32] H. Mughrabi, Microstructural fatigue mechanisms: Cyclic slip irreversibility, crack initiation, nonlinear elastic damage analysis Int. J. Fatigue 57 (2013) 2-8.

[33] N. Thompson, Dislocation Nodes in Face-Centred Cubic Lattices, Proc. Phys. Soc. B 66 (6) (1953) 481-492.

[34] L. Brown, Dislocation plasticity in persistent slip bands, Mater. Sci. Eng. A 285 (1-2) (2000) 35-42.

[35] U. Essmann, H. Mughrabi, Annihilation of dislocations during tensile and cyclic deformation and limits of dislocation densities Philos. Mag. A 40 (6) (1979) 731-756.

[36] B. Escaig, Sur le glissement dévié des dislocations dans la structure cubique à faces centrées J. Phys. 29 (2-3) (1968) 225-239.

[37] K. Kang, J. Yin, W. Cai, Stress dependence of cross slip energy barrier for face-centered cubic nickel, J. Mech. Phys. Solids 62 (2014) 181-193.

[38] A. Malka-Markovitz, D. Mordehai, Cross-slip in face-centered cubic metals: a general Escaig stressdependent activation energy line tension model Philos. Mag. 98 (5) (2018) 347-370.

[39] C. Huvier, E. Conforto, H. E. Alami, D. Delafosse, X. Feaugas, Some correlations between slip band emergence and dislocation pattern IOP Conf. Ser.: Mater. Sci. Eng. 3 (2009) 012012.

[40] K. Obrtlík, M. Juliš, J. Man, T. Podrábský, J. Polák, Extrusion and intrusion evolution in cyclically strained cast superalloy Inconel 738LC using confocal laser scanning microscope and AFM J. Phys.: Conf. Ser. 240 (2010) 012054.

[41] H. S. Ho, M. Risbet, X. Feaugas, G. Moulin, Damage mechanism related to localization of plastic deformation of Waspaloy: Effect of grain size, Procedia Eng. 10 (2011) 863-868.

[42] T. Zhang, J. Jiang, B. Britton, B. Shollock, F. Dunne, Crack nucleation using combined crystal plasticity modelling, high-resolution digital image correlation and high-resolution electron backscatter diffraction in a superalloy containing non-metallic inclusions under fatigue Proc. R. Soc. A $472(2189)$ (2016) 1-25.

[43] F. Bourdin, J. Stinville, M. Echlin, P. Callahan, W. Lenthe, C. Torbet, D. Texier, F. Bridier, J. Cormier, P. Villechaise, T. Pollock, V. Valle, Measurements of plastic localization by heavisidedigital image correlation, Acta Mater. 157 (2018) 307-325. 


\section{Appendix A. Slip line characterisation technique from $\gamma^{\prime}$-etched samples}

The following methodology is based upon the identification of a number of features that can be observed in micrographs where the precipitates in a plastically deformed sample have been etched away. An example of such a micrograph is shown in Figure 1(a) with highlighted slip lines. The applicability of this method depends upon the original size and morphology of these particles, the error being less for larger and more spherical precipitates.

The aim of this technique is to find the rotation angles a sheared particle must undergo to match the shape of the observed cavities. The relevant angles and rotation axes are: $\phi$ around the sample surface normal, $\alpha$ around the slip line projection and $\beta$ around the slip plane normal. Of these, $\phi$ can be directly obtained from the micrograph by measuring the angle between a horizontal line and the traced slip lines.

To define the directions and angles required to describe the orientation and real distances of interest, consider three orthogonal right-handed coordinate systems: $(x, y, z)$ with the $x$ and $y$ axes aligned with the sides of the micrograph, $\left(x^{\prime}, y^{\prime}, z^{\prime}\right)$ resulting from the rotation along the $z$ axis by angle $\phi$ so that the active slip markings are parallel to $x^{\prime}$, and $\left(x^{\prime \prime}, y^{\prime \prime}, z^{\prime \prime}\right)$ with $x^{\prime \prime}=x^{\prime}, y^{\prime \prime}$ parallel to the slip plane normal and positioned at the centre of the precipitate on the shear plane. These coordinate systems can be seen in Figures A.16(a) and (b) along with other parameters described in this section.

(a)

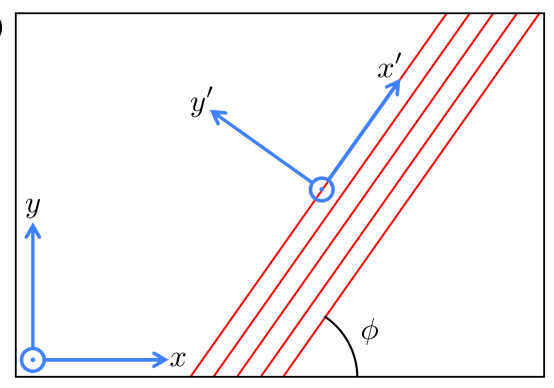

(c)

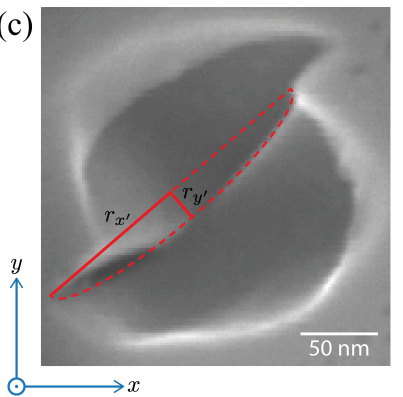

(d) (b)
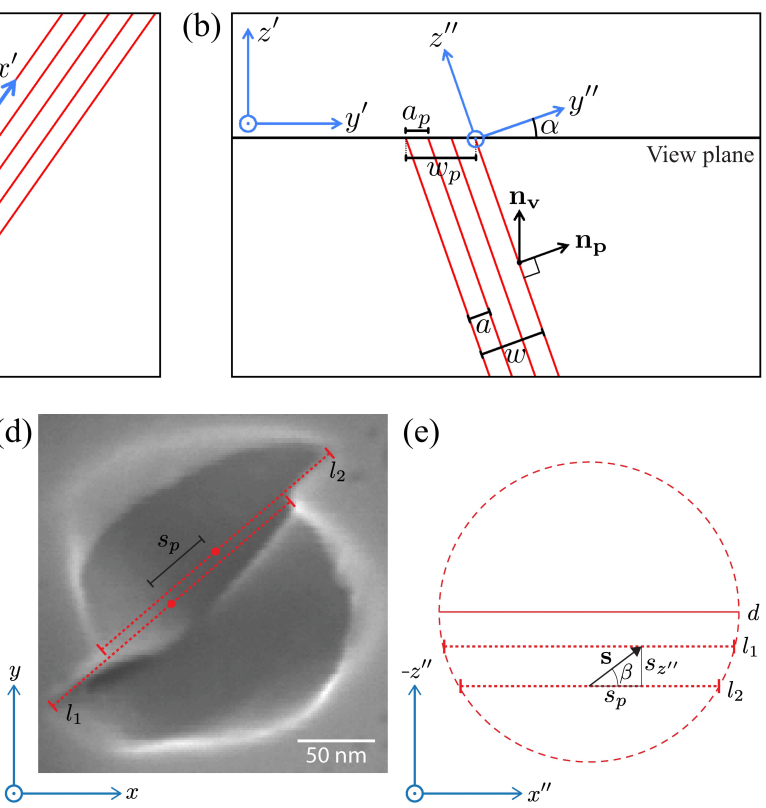

(e)

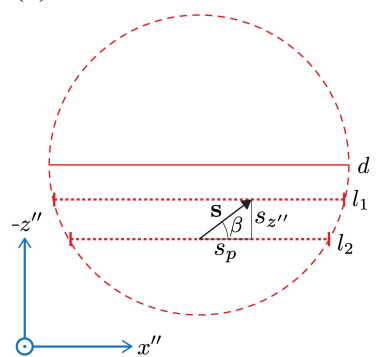

Figure A.16: Schematic representations of the coordinate systems (blue) and slip lines (red) as seen from (a) the direction of the electron beam and (b) a lateral view with the slip band edge-on. Lengths required to calculate (c) $\alpha$ and (d) $\beta$ from a precipitate hole and (e) precipitate cross section on the slip plane where shearing occurs in the direction $\mathbf{s}$.

Sheared holes show the presence of elliptical marks. These are the projections onto the viewing plane of the circular cross sections along which shearing of the precipitates occur. 
Assuming an initial perfect sphericity, it would be possible to unequivocally determine the value of the angle $\alpha$ by measuring the ratio between the major and minor radii of the ellipse, $r_{x^{\prime}}$ and $r_{y^{\prime}}$ respectively, as

$$
\alpha=\arcsin \left(\frac{r_{y^{\prime}}}{r_{x^{\prime}}}\right) .
$$

These features are drawn for a precipitate in Figure A.16(c) as an example.

To calculate $\beta$ consider a vector in the third coordinate system $\mathbf{s}=\left(s_{p}, 0, s_{z^{\prime \prime}}\right)$ defining the direction and magnitude of a shear step. It is possible to directly measure $s_{p}$ as the centre-to-centre distance between both halves of the sheared hole. Due to the in- and out-of-plane component of the shear direction, the segments along the slip line of the top and bottom halves of the hole $l_{1}$ and $l_{2}$ will not have the same length. The $z^{\prime \prime}$-coordinates of such segments before shearing occurred can be calculated from

$$
z_{i}^{\prime \prime}=\frac{1}{2} \sqrt{d^{2}-l_{i}^{2}}
$$

where $d$ is the diameter of the cross section at the shear plane. This distance can be detected and measured even tenths of nanometers under the surface in cases where more than half of the precipitate was positioned below the specimen surface thanks to the high voltage of the electron beam. Moreover, $s_{z^{\prime \prime}}=\left|z_{2}^{\prime \prime}-z_{1}^{\prime \prime}\right|$ and

$$
\beta=\arctan \left(\frac{s_{z^{\prime \prime}}}{s_{p}}\right) .
$$

Figure A.16(d) shows an example of the aforementioned lengths. A schematic diagram of the cross section on the slip plane is drawn in Figure A.16(e) before shearing occurs, showcasing the origin of $\beta$.

The angles $\alpha$ and $\beta$ are the only parameters required to access the true morphology of the slip bands and shearing distances at the multiple slip lines observed. The $y^{\prime \prime}$ axis is relevant as both the slip band width $w$ and slip line spacing $a$ lengths lie along this direction. From figure A.16(b) it can readily be seen that

$$
a=a_{p} \cos \alpha
$$

where $a_{p}$ is the projection of $a$ onto the $\left(x^{\prime}, y^{\prime}\right)$ plane and $w=\sum_{i} a_{i}$ for each slip band. Similarly, the true shear step lengths $s$ at each slip line are

$$
s=\frac{s_{p}}{\cos \beta} .
$$

The Schmid factor $m_{S}$ can also be calculated if the direction of unidirectional loading $\mathbf{n}_{\boldsymbol{\sigma}}$ is known. If $R_{1}$ and $R_{2}$ denote the rotation matrices by angles $\phi$ and $\alpha$, the components of the active slip system in the $(x, y, z)$ coordinate system can be extracted via $\mathbf{n}_{\mathbf{p}}=R_{1}^{-1} R_{2}^{-1}[0,1,0]^{T}$ and $\mathbf{n}_{\mathbf{s}}=R_{1}^{-1} R_{2}^{-1}[\cos \beta, 0, \sin \beta]^{T}$, where $n_{s}$ is the unit vector in the direction of the shear steps. The Schmid factor is then $m_{S}=\left(\mathbf{n}_{\boldsymbol{\sigma}} \cdot \mathbf{n}_{\mathbf{p}}\right)\left(\mathbf{n}_{\boldsymbol{\sigma}} \cdot \mathbf{n}_{\mathbf{s}}\right)$. Even though information regarding the crystal is obtained with this technique, it is impossible to fully determine the orientation of other slip planes as two mirrored Thompson's tetrahedrons may contain a slip system with the orientation found. 